\title{
Embryology of a melanoma? A case report with speculation based on dermatoscopic and histologic evidence
}

\author{
Cliff Rosendahl MBBS ${ }^{1}$, Alan Cameron $\mathrm{MBBS}^{1}$, Agata Bulinska M.D. ${ }^{1}$, \\ David Harding-Smith MBBS$^{2}$, David Weedon, M.D. ${ }^{3}$
}

\footnotetext{
${ }^{1}$ School of Medicine, The University of Queensland, Brisbane, Australia

${ }^{2}$ Central Medical Bundaberg, Bundaberg, Australia

${ }^{3}$ Sullivan Nicolaides Pathology, Brisbane, Australia
}

Key words: melanoma, nevus, small melanoma, histologic criteria, dermatoscopy

Citation: Rosendahl C, Cameron A, Bulinska A, Harding-Smith D, Weedon D. Embryology of a melanoma? A case report with speculation based on dermatoscopic and histologic evidence. Dermatol Pract Conc. 2012;2(4):08. http://dx.doi.org/10.5826/dpc.0204a08.

Received: May 9, 2012; Accepted: July 31, 2012; Published: October 31, 2012

Copyright: @2012 Rosendahl et al. This is an open-access article distributed under the terms of the Creative Commons Attribution License, which permits unrestricted use, distribution, and reproduction in any medium, provided the original author and source are credited.

Funding: None.

Competing interests: The authors have no conflicts of interest to disclose.

All authors have contributed significantly to this publication.

Corresponding author: Cliff Rosendahl, PO Box 734, Capalaba, Queensland, Australia 4157. Tel. +61.7.3245.3011; Fax.+61.7.3245.3022. Email: cliffrosendahl@bigpond.com.

\section{From The Lives of Lesions}

"The idea evolved that lesions have lives, just as human beings have; that lesions look very different at different times in their lives just as human beings do; and that stages in the lives of lesions can be described and depicted roughly as early, fully developed, and late, just as human beings can be described and depicted as infantile, mature, and old."

A. Bernard Ackerman, M.D.

From The Lives of Lesions, page vi (Ardor Scribendi, Ltd, 1983)

\section{Introduction}

A dermatopathologist can only make a specific diagnosis based on the presence of defined histologic criteria. Most cases encountered in routine practice show enough criteria on standard examination to reach an unequivocal diagnosis. Sometimes further steps must be taken, such as cutting deeper levels or using special stains, to clarify uncertainties. Occasionally the final histologic diagnosis must remain uncertain or borderline. This most frequently occurs in the assessment of melanocytic lesions.

There is no single diagnostic criterion for the histologic diagnosis of melanoma. "Criteria are evaluated differently by different observers. They are derived from typical, not difficult cases, and are seldom tested. In sum, none of the criteria useful for the diagnosis of melanoma are specific and diagnostic ..." [1].

A series of architectural and cytological criteria are listed (Table 1) [2] and these criteria would apply to the majority of melanomas encountered in routine dermatopathology practice, but the quantity or quality required to make a diagnosis are not defined. It is accepted that melanomas, as with all malignant tumours, evolve over time and the rate of growth has been measured in one study [3]. Currently, few very small melanomas are diagnosed [4,5]. The increased use of dermatoscopy and digital monitoring should lead to 
TABLE 1. Histologic criteria for the diagnosis of malignant melanoma (after Ackerman) (Table 32.4, Weedon's Skin Pathology [2].)

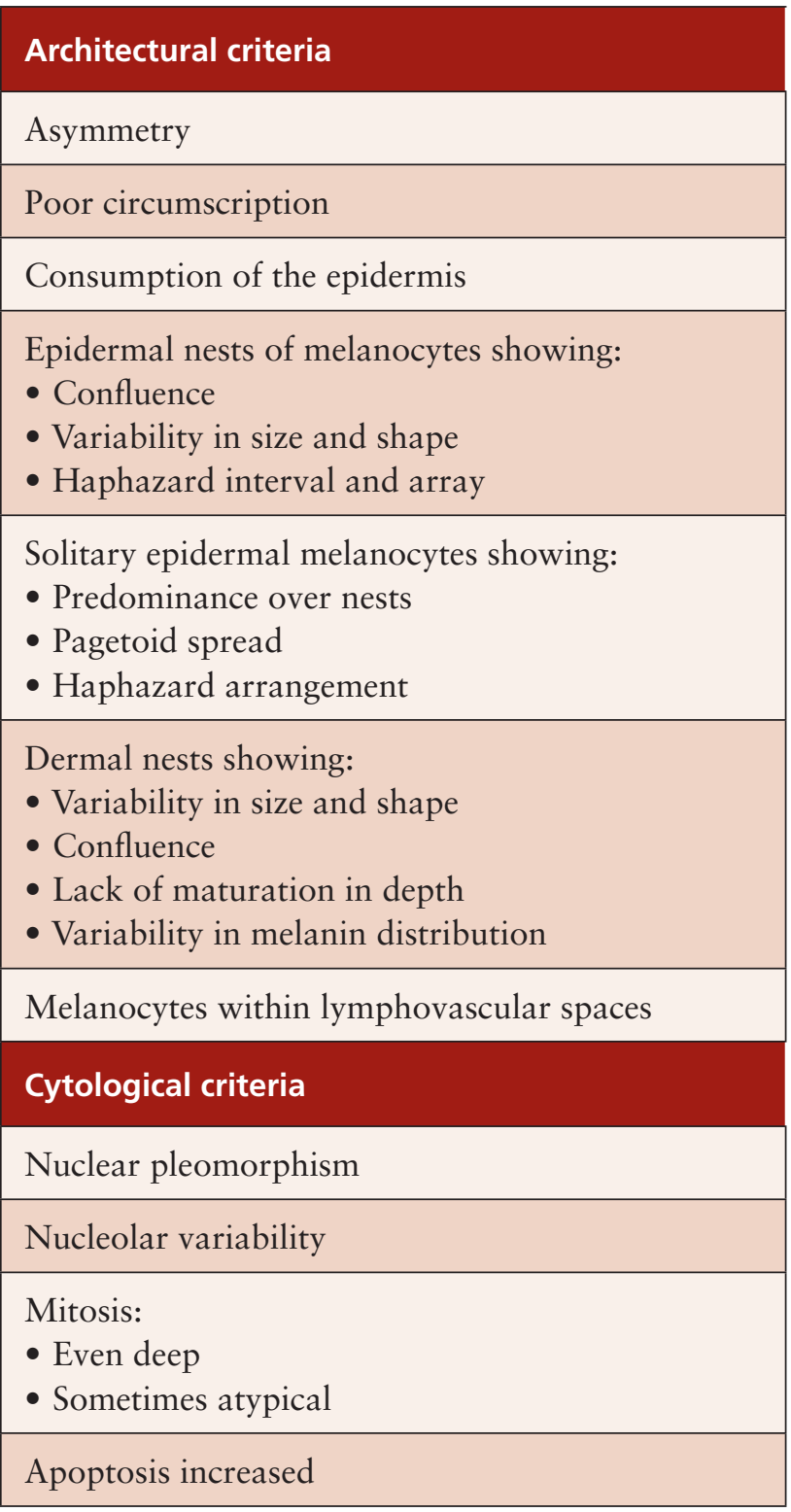

more small melanomas being encountered [6]. What is not known is how commonly these very small melanomas, at the 'infantile' stage of Ackerman's analogy, lack sufficient criteria to make the diagnosis of melanoma. It seems reasonable to speculate that such lesions exist. This leads us to pose the question, "Can melanomas be diagnosed earlier by adding information from history and dermatoscopy to the findings at histopathology?" and we present the case that led us to consider these matters.

\section{Case report}

A 51-year-old man with a history of five previously treated melanomas was referred for a skin examination. Two lesions were identified as clinically and dermatoscopically suspicious for melanoma and were excised. They were both subsequently signed out as melanomas, one (on the back) invasive and one (on the calf) in-situ. As he was rising from the operating table the patient indicated a $2 \mathrm{~mm}$ pigmented lesion on his face (Figure 1). He stated that he had been thinking about the doctor's earlier question about whether he had noticed any new or changing skin lesions and he stated that he had only been aware of this lesion for two weeks. He was quite certain about this and stated that this was because of his heightened awareness of his risk for melanoma based on his past history.

Dermatoscopic assessment (Figure 2) revealed a brown structureless lesion with several discrete gray circles, some complete and some incomplete (asymmetrically pigmented). Gray structures, and more specifically gray circles, have been described as a clue to melanoma $[7,8]$ correlating with extension of melanin-containing melanoma cells down hair follicles, and asymmetrically pigmented follicular openings are described as a clue to lentigo maligna [9]. Furthermore it has been recommended that the presence of any dermatoscopic gray structures on the face should lead to a biopsy [6].
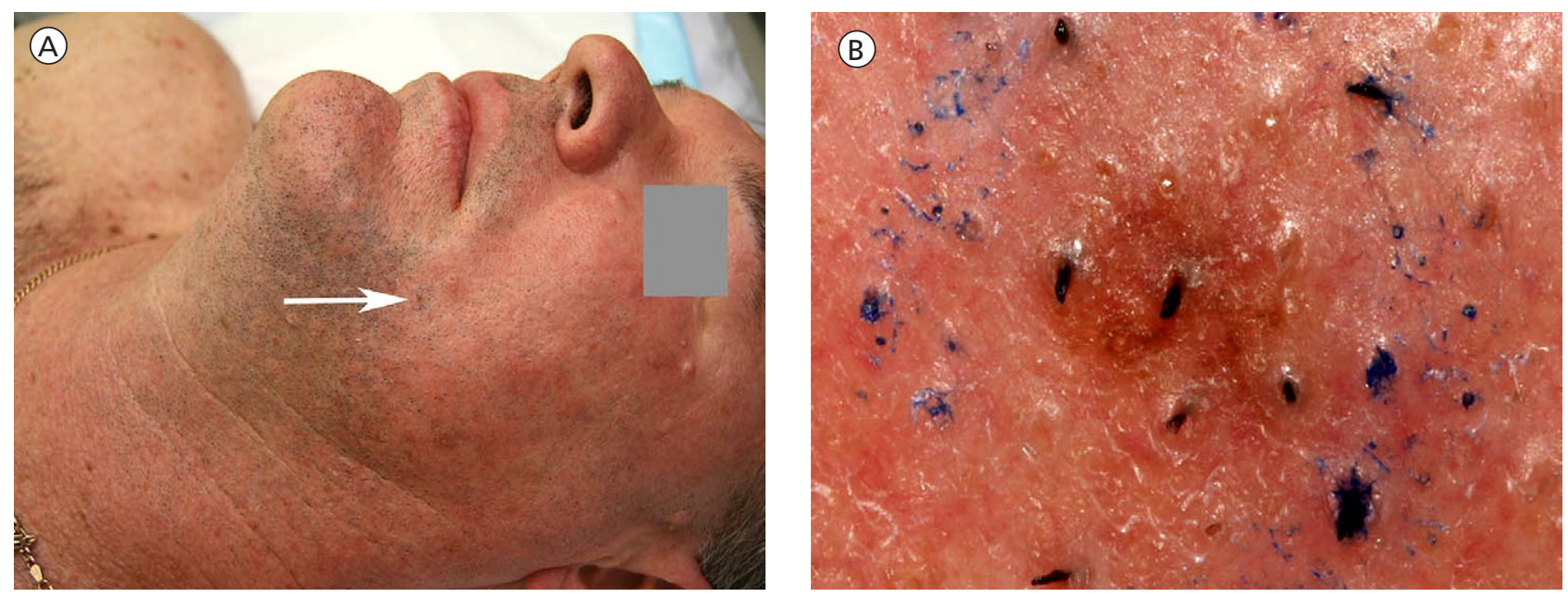

Figure 1. Clinical and close-up image of a (reportedly new) $2 \mathrm{~mm}$ pigmented skin lesion on the face of a 51-year-old man. [Copyright: (02012 Rosendahl et al.] 


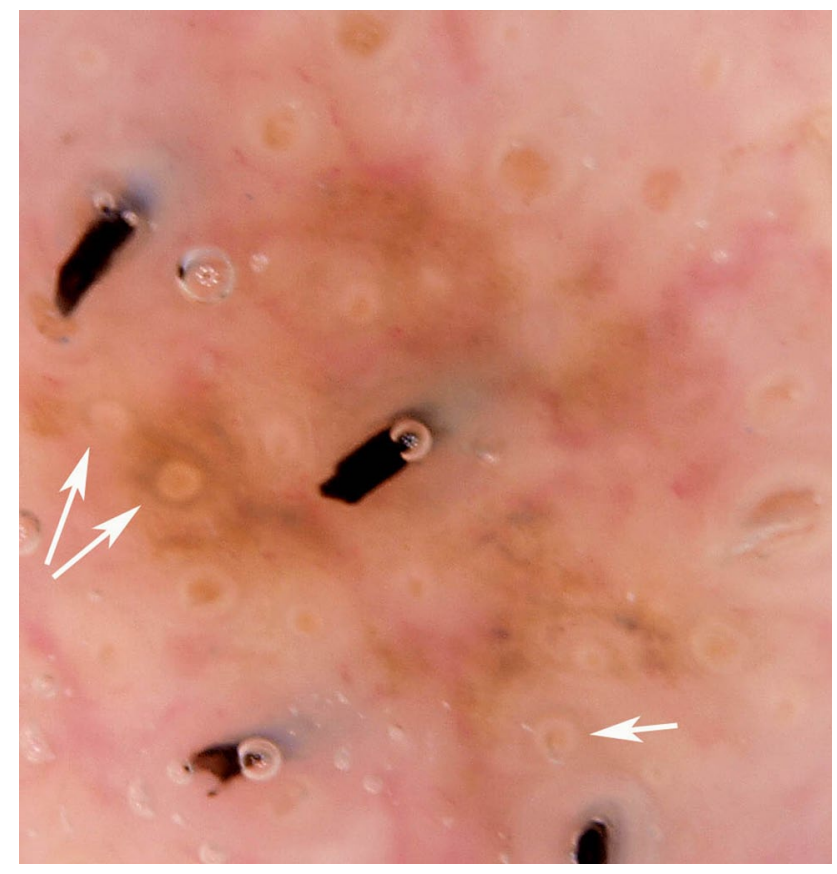

Figure 2. A dermatoscopic image reveals the presence of gray circles (three of which are indicated by arrows). [Copyright: @2012 Rosendahl et al.]

An excision biopsy was performed and histologic examination (Figures 3-5) revealed a melanocytic proliferation including areas with a lentiginous array of single melanocytes as well as some nesting at the tips of rete ridges, with some melanocytes, although small, exhibiting pleomorphic and hyperchromatic nuclei. There was a very focally confluent proliferation of single melanocytes extending down two follicles.

The reporting pathologist (author DW) was unable to make a diagnosis of melanoma based on currently accepted criteria. This was not based on the absence of any criteria for melanoma but rather on an insufficient degree of cytological and architectural atypia. The pathologist did agree that there were certain apparent inconsistencies with the alternative diagnosis of nevus:

A new (macular) junctional nevus is uncommon on the central part of the face at mature age. (Personal observation by author DW.)

Although it is seen occasionally, extension down a follicle is uncommon in a non-congenital-type nevus. (Personal observation by author DW.)

However a diagnosis of "atypical nevus" was issued and this was confirmed by another senior pathologist at the same institution.

\section{Conclusion}

We believe that the diagnosis of melanoma at an early stage is desirable. With this in mind we argue that clinical and

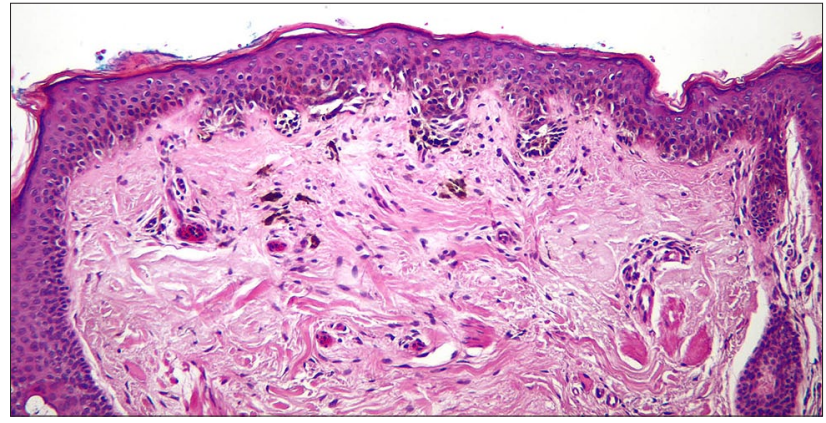

Figure 3. Low power view of the pigmented skin lesion illustrated above showing a melanocytic proliferation with some lentiginous array of single melanocytes as well as nesting. On the left a lentiginous array of single melanocytes extending down a hair follicle is clearly seen. [Copyright: (C2012 Rosendahl et al.]

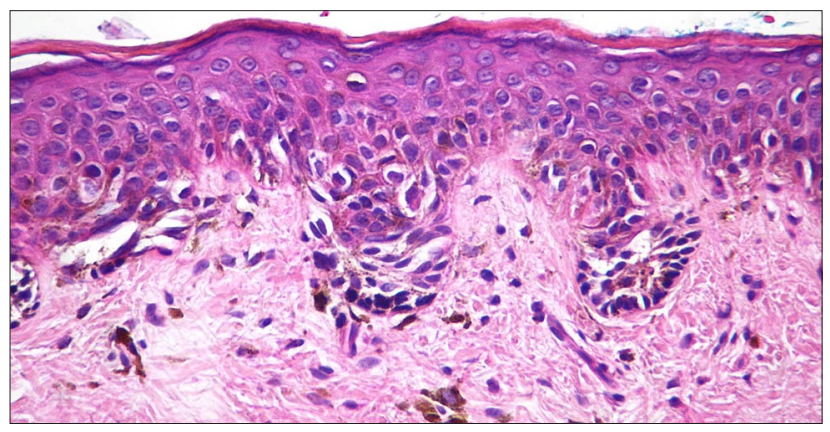

Figure 4. Higher power view showing nesting of (small) melanocytes with pleomorphic hyperchromatic nuclei and apparent partial thickness Pagetoid spread. [Copyright: @2012 Rosendahl et al.]

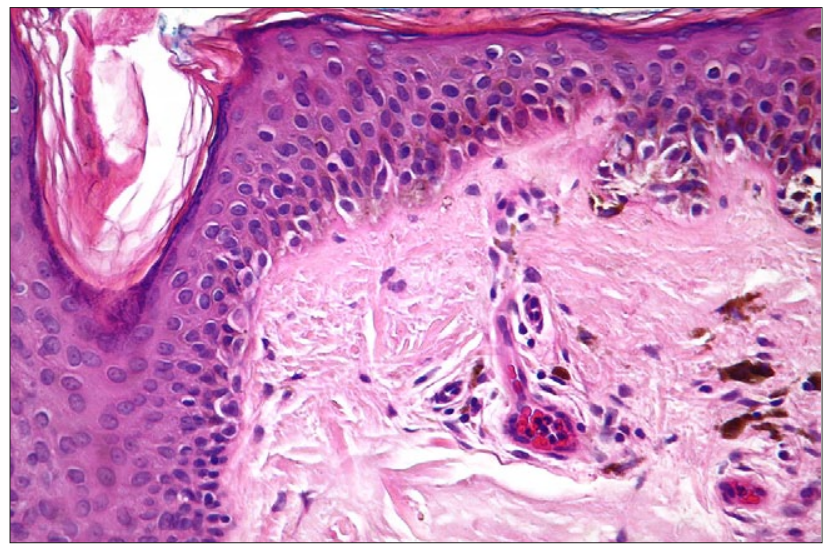

Figure 5. Higher power view of focally confluent lentiginous single melanocytes extending into the superficial part of a follicle. [Copyright: (02012 Rosendahl et al.]

dermatoscopic clues that support the diagnosis of melanoma should be communicated to the reporting pathologist, prompting a more careful evaluation of histologic features in very small melanocytic lesions. Furthermore, using the analogy of Ackerman, we suggest that in order not to misdiagnose 'infantile' melanomas, the diagnosis of melanoma should be entertained at a lower threshold in very small lesions, when borderline criteria are supported by clinical and dermatoscopic clues. 


\section{References}

1. Massi G, Leboit PE. Histological Diagnosis of Nevi and Melanoma. Darmstadt, Germany: Steinkopff Verlag, 2004.

2. Weedon D. Weedon's Skin Pathology. 3rd ed. London, England: Churchill Livingstone Elsevier, 2010.

3. Beer J, Xu L, Tschandl P, Kittler H. Growth rate of melanoma in vivo and correlation with dermatoscopic and dermatopathologic findings. Dermatol Pract Conc. 2011;1(1):13. http://dx.doi. org/10.5826/dpc.0101a13.

4. Abbasi NR, Shaw HM, Rigel DS, et al. Early diagnosis of cutaneous melanoma: revisiting the ABCD criteria. JAMA. 2004;292(22):2771-6.

5. Teng PP, Hofmann-Wellenhof R, Campbell TM, Soyer HP. Dermoscopic presentation of a 2-mm melanoma in situ. Australas J Dermatol. 2010;51(2):152-3.
6. Rosendahl C, Cameron A, Bulinska A, Williamson R, Kittler H. Dermatoscopy of a minute melanoma. Australas J Dermatol. 2011;52(1):76-8.

7. Rosendahl C, Tschandl P, Cameron A, Kittler H. Diagnostic accuracy of dermatoscopy for melanocytic and nonmelanocytic pigmented lesions. J Am Acad Dermatol. 2011;64(6):1068-73.

8. Rosendahl C, Cameron A, Tschandl P, Williamson R, Kittler H. The Cause of Dermatoscopic Grey Circles in Non-Invasive Melanomas: An Hypothesis Supported by Histopathological Correlation. Poster presented at the 2nd Congress of the International Dermoscopy Society, 2009, November 12th -14th, Barcelona, Spain. www.greycircles.blogspot.com accessed 28/9/2010 at 12:00 GMT.

9. Schiffner R, Schiffner-Rohe J, Vogt T, et al. Improvement of early recognition of lentigo maligna using dermatoscopy. J Am Acad Dermatol. 2000;42(1 Pt 1):25-32. 